\title{
Application of Perturbation Theory to a Master Equation
}

\author{
B. M. Villegas-Martínez, F. Soto-Eguibar, and H. M. Moya-Cessa \\ Instituto Nacional de Astrofísica, Óptica y Electrónica, INAOE, Calle Luis Enrique Erro 1, \\ 72840 Santa María Tonantzintla, PUE, Mexico \\ Correspondence should be addressed to F. Soto-Eguibar; feguibar@inaoep.mx
}

Received 16 March 2016; Accepted 17 May 2016

Academic Editor: Antonio Scarfone

Copyright (c) 2016 B. M. Villegas-Martínez et al. This is an open access article distributed under the Creative Commons Attribution License, which permits unrestricted use, distribution, and reproduction in any medium, provided the original work is properly cited.

\begin{abstract}
We develop a matrix perturbation method for the Lindblad master equation. The first- and second-order corrections are obtained and the method is generalized for higher orders. The perturbation method developed is applied to the problem of a lossy cavity filled with a Kerr medium; the second-order corrections are estimated and compared with the known exact analytic solution. The comparison is done by calculating the $Q$-function, the average number of photons, and the distance between density matrices.
\end{abstract}

\section{Introduction}

Open systems, that is, systems that interact with an environment, represent an important problem in many branches of physics such as cosmology [1-3], quantum information [4], quantum optics [5], and condensed matter [6]. The irreversible loss of information and the dissipative process generated by a reservoir are a sign that the environment plays an important role in the dynamics of physical systems $[5,7,8]$. Open system dynamics are often determined by an equation of motion for the density matrix, the master equation. Usually these equations require certain assumptions to provide the dynamic description [9-12]. The Lindblad master equation represents one of the simplest cases that describe losses in an adequate form. Unfortunately, even this master equation is difficult to treat and to solve in an exact form [13-15]; hence, it is often required to apply a perturbative treatment. Many perturbative methods have been developed to solve particular problems modeled by the Lindblad master equation, such as a two-level nonlinear quantum system, a single-mode field in a lossy cavity, two-level atom coupling to a Bose-mode environment, and a single atom coupling to a mode of a lossy cavity [16-18]. In fact, it has been shown that even though decoherence takes place, the reconstruction of quasiprobability distribution functions may be achieved in atomfield $[19,20]$ or laser-trapped ion interactions [21].

In this work, we show that it is possible to implement a matrix perturbation method on the Lindblad master equation that allows us to determine in a simple and effective form the $m$ th-order correction. The present paper is divided into two parts. In the first one, we develop a perturbative method for the Lindblad master equation by using superoperator techniques in terms of matrices; we obtain the first- and secondorder corrections; these results lead to the generalization of the method to further order corrections. In the second part, the validity of the method is verified through a particular problem concerning a lossy cavity filled with a Kerr medium that has exact analytical solution. We obtain an approximate solution using the method proposed in this contribution and compare it with the exact result. The verification of both results is based on a comparative evaluation of the system via $Q$-function, the average photon number, and the measure of the distance between its density matrices.

\section{Lindblad Master Equation}

The Lindblad master equation, which describes the interaction between a given system and its environment at zerotemperature, is given by $[5,6,22]$

$$
\frac{d \rho}{d t}=(\widehat{S}+\lambda \widehat{\mathscr{L}}) \rho
$$

In this equation, $\rho$ is the density matrix; the superoperator $\widehat{S}$ is (we have set $\hbar=1$ )

$$
\widehat{S} \rho=-i[H, \rho]
$$


with $H$ being the interaction (time-independent) Hamiltonian; the superoperator $\widehat{\mathscr{L}}$, which describes the interaction of the system with the environment, is given by

$$
\widehat{\mathscr{L}} \rho=\gamma\left(2 a \rho a^{\dagger}-a^{\dagger} a \rho-\rho a^{\dagger} a\right),
$$

with $a$ and $a^{\dagger}$ being the usual creation and annihilation operators and $\gamma$ is the rate at which the system loses energy; and, finally, $\lambda$ is a perturbation parameter (effectively, the perturbation parameter is $\lambda \gamma$ as we consider small $\gamma$ 's).

The formal solution to the master equation is

$$
\rho(t)=e^{(\widehat{S}+\lambda \widehat{\mathscr{L}}) t} \rho(0),
$$

where $\rho(0)$ is the density matrix of the initial state of the system.

2.1. First-Order Correction. To get the first-order correction to the nonperturbed solution to the master equation, we expand the exponential in (4) in Taylor series and keep only first-order terms in $\lambda$ :

$$
\rho(t) \approx\left(\sum_{n=0}^{\infty} \frac{t^{n}}{n !} \widehat{S}^{n}+\lambda \sum_{n=1}^{\infty} \frac{t^{n}}{n !} \sum_{m=0}^{n-1} \widehat{S}^{m} \widehat{\mathscr{L}}^{n-m-1}\right) \rho(0) .
$$

We simplify the above expression using the matrix method [23]; we define a triangular $2 \times 2$ array of superoperators, where the diagonal elements are given by the nonperturbed system and the superior triangle contains the perturbation:

$$
M \rho=\left(\begin{array}{cc}
\widehat{S} & \widehat{\mathscr{L}} \\
0 & \widehat{S}
\end{array}\right) \rho .
$$

Equation (5) can then be written as

$$
\rho(t) \approx\left(e^{\hat{S} t}+\lambda\left(e^{M t}\right)_{1,2}\right) \rho(0),
$$

where $\left(e^{M t}\right)_{1,2}$ stands for the element $(1,2)$ of the matrix $e^{M t}$. In what follows, we will denote the matrix elements of a matrix by a couple of integers subindexes. In this expression, we have separated the first-order approximation into two parts; one involves only the system and the other tells us how the environment affects the system. Thus, we can split the density matrix into one part concerning the nonperturbed system and a small contribution in terms of $\lambda$ referent to firstorder perturbation; so we can write (7) as

$$
\rho(t) \approx \rho^{(0)}(t)+\lambda\left(\rho^{P}\right)_{1,2},
$$

where $2 \times 2$ perturbed density matrix is given by

$$
\rho^{P}=\left(\begin{array}{ll}
\rho_{1,1} & \rho_{1,2} \\
\rho_{2,1} & \rho_{2,2}
\end{array}\right) .
$$

Deriving (7) and (8) with respect to time and equating $\lambda$ terms, we obtain the differential equation

$$
\frac{d}{d t}\left(\rho^{P}\right)_{1,2}=\left(M e^{M t} \rho(0)\right)_{1,2}
$$

or the equivalent systems of differential equations

$$
\frac{d}{d t}\left(\begin{array}{ll}
\rho_{1,1} & \rho_{1,2} \\
\rho_{2,1} & \rho_{2,2}
\end{array}\right)=M e^{M t}\left(\begin{array}{cc}
\rho(0) & 0 \\
0 & \rho(0)
\end{array}\right) .
$$

This system of equations can be solved and it is easy to see that the differential equation

$$
\frac{d}{d t} \rho^{P}=M \rho^{P}
$$

is also satisfied. By doing a matrix multiplication in the above expression, we can prove that the first-order solution is related to the second column of perturbed density matrix; that is,

$$
\frac{d}{d t}\left(\begin{array}{c}
\rho_{1,2} \\
\rho_{2,2}
\end{array}\right)=M\left(\begin{array}{c}
\rho_{1,2} \\
\rho_{2,2}
\end{array}\right) .
$$

Doing the transformation $\phi_{1,2}=e^{-\widehat{S} t} \rho_{1,2}$, we have

$$
\frac{d}{d t} \phi_{1,2}=e^{-\widehat{S} t} \widehat{\mathscr{L}} e^{\widehat{S} t} \rho(0)
$$

and so

$$
\rho_{1,2}=e^{\widehat{S} t}\left[\int_{0}^{t} e^{-\widehat{S} t_{1}} \widehat{\mathscr{L}} e^{\widehat{S} t_{1}} d t_{1}\right] \rho(0)
$$

2.2. Second-Order Correction. The second-order correction to the nonperturbed solution to the master equation may be obtained if we take into account the terms in $\lambda^{2}$ from the Taylor series expansion (5); we have then

$$
\begin{aligned}
\rho(t) \approx & \sum_{n=0}^{\infty} \frac{t^{n}}{n !} \widehat{S}^{n} \rho(0)+\lambda \sum_{n=1}^{\infty} \frac{t^{n}}{n !} \sum_{m=0}^{n-1} \widehat{S}^{m} \widehat{\mathscr{L}} \widehat{S}^{n-m-1} \rho(0) \\
& +\lambda^{2} \sum_{n=2}^{\infty} \frac{t^{n}}{n !} \sum_{m=1}^{n-1} \sum_{j=0}^{n-m-1} \widehat{S}^{n-m-j-1} \widehat{\mathscr{L}} \widehat{S}^{j} \widehat{\mathscr{L}}^{m-1} \rho(0)
\end{aligned}
$$

In this case, we increase the dimension of our superoperators matrix as follows:

$$
M \rho=\left(\begin{array}{ccc}
\widehat{S} & \widehat{\mathscr{L}} & 0 \\
0 & \widehat{S} & \widehat{\mathscr{L}} \\
0 & 0 & \widehat{S}
\end{array}\right) \rho .
$$

In the previous section, it is demonstrated that the element $(1,2)$ of $M^{n}$ gave us all terms of the first-order correction. The second order will be a similar situation: all the information will be in the element $(1,3)$ of the new $M$ raised to the power $n$; indeed, we get

$$
\rho(t) \approx\left[e^{\widehat{S} t}+\lambda\left(e^{M t}\right)_{1,2}+\lambda^{2}\left(e^{M t}\right)_{1,3}\right] \rho(0) .
$$


Following the same steps that are in the first-order case, we can write the density matrix as

$$
\rho(t) \approx \rho^{(0)}(t)+\lambda\left(\rho^{(P)}\right)_{1,2}+\lambda^{2}\left(\rho^{(P)}\right)_{1,3}
$$

where the solution to $\rho_{1,3}$ will be associated with the third column of the perturbed density matrix

$$
\frac{d}{d t}\left(\begin{array}{c}
\rho_{1,3} \\
\rho_{2,3} \\
\rho_{3,3}
\end{array}\right)=M\left(\begin{array}{c}
\rho_{1,3} \\
\rho_{2,3} \\
\rho_{3,3}
\end{array}\right) .
$$

Solving the system of equations through the transformations $\phi_{1,3}=e^{-\widehat{S} t} \rho_{1,3}$ and $\phi_{2,3}=e^{-\widehat{S} t} \rho_{2,3}$, we get the second-order correction

$$
\rho_{1,3}=e^{\widehat{S} t} \int_{0}^{t} \int_{0}^{t_{1}} e^{-\widehat{S} t_{1}} \widehat{\mathscr{L}} e^{\widehat{S} t_{1}} e^{-\widehat{S} t_{2}} \widehat{\mathscr{L}} e^{\widehat{S} t_{2}} d t_{2} d t_{1} \rho(0) .
$$

2.3. Higher Orders. The generalization of the method for higher-order corrections can be obtained directly from the results of the first-order and second-order corrections. So, following the same steps that take us to expression (21), we define the semi-infinite superoperators array:

$$
M=\left(\begin{array}{ccccc}
\widehat{S} & \widehat{\mathscr{L}} & \cdots & 0 & 0 \\
0 & \widehat{S} & \widehat{\mathscr{L}} & \cdots & 0 \\
\vdots & 0 & \widehat{S} & \ddots & \vdots \\
\vdots & 0 & 0 & \ddots & \widehat{\mathscr{L}} \\
0 & \vdots & 0 & 0 & \widehat{S}
\end{array}\right),
$$

and the $m$ th-order correction can be expressed as

$$
\begin{aligned}
\rho_{1, m+1}= & e^{\widehat{S} t} \int_{0}^{t} \int_{0}^{t_{1}} \cdots \int_{0}^{t_{m}-1} e^{-\widehat{S} t_{1}} \widehat{\mathscr{L}} e^{\widehat{S} t_{1}} e^{-\widehat{S} t_{2}} \widehat{\mathscr{L}} e^{\widehat{S} t_{2}} \cdots \\
& -e^{-\widehat{S} t_{m}} \widehat{\mathscr{L}} e^{\widehat{S} t_{m}} d t_{m} \cdots d t_{2} d t_{1} \rho(0)
\end{aligned}
$$

\section{Lossy Cavity Filled with a Kerr Medium}

To demonstrate the accuracy and capability of the method, we obtain the perturbative solution to the master equation of a Kerr medium filling an optical cavity with losses. The exact analytic solution for the master equation in this case is [24]

$$
\rho_{\text {exa }}(\tau)=e^{\widehat{S} \tau} e^{\widehat{L} \tau} \exp \left[\frac{1-e^{-2 \tau(\lambda+i \widehat{R})}}{2(\lambda+i \widehat{R})} \widehat{J}\right] \rho(0),
$$

with $\tau=\chi t, \lambda=\gamma / \chi$, and the parameter $\lambda$ being the ratio between the cavity decay and the Kerr medium constant. The superoperators $\widehat{S}, \widehat{J}, \widehat{L}$, and $\widehat{R}$ are defined as

$$
\begin{aligned}
& \widehat{S} \rho=-i\left[a^{\dagger 2} a^{2}, \rho\right], \\
& \widehat{J} \rho=2 \lambda a \rho a^{\dagger}, \\
& \widehat{L} \rho=-\lambda\left(a^{\dagger} a \rho+\rho a^{\dagger} a\right), \\
& \widehat{R} \rho=a^{\dagger} a \rho-\rho a^{\dagger} a,
\end{aligned}
$$

and they satisfy the commutation relations

$$
\begin{aligned}
& {[\widehat{S}, \widehat{J}] \rho=2 i \widehat{R} \widehat{J} \rho,} \\
& {[\widehat{L}, \widehat{J}] \rho=2 \lambda \widehat{J} \rho,} \\
& {[\widehat{R}, \widehat{J}] \rho=0 .}
\end{aligned}
$$

As initial state condition of the system, one can assume a coherent state; that is, $\rho(0)=|\alpha\rangle\langle\alpha|$; so, with the help of the commutation relations, one gets

$$
\rho_{\text {exa }}(\tau)=e^{-|\alpha|^{2}} \sum_{n=0}^{\infty} \sum_{m=0}^{\infty} C_{n, m}|n\rangle\langle m|,
$$

where

$$
C_{n, m}=\frac{\alpha^{n} \alpha^{* m}}{\sqrt{n ! m !}} e^{-i[n(n-1)-m(m-1)] \tau} D_{n, m}
$$

and with

$$
D_{n, m}=e^{-\lambda(n+m) \tau} \exp \left[\lambda|\alpha|^{2} \frac{1-e^{-2 \tau(\lambda+i(n-m))}}{\lambda+i(n-m)}\right] .
$$

The exact density matrix shows how the initial coherent state structure is lost due to dissipation of energy generated by the cavity walls and the quadratic terms associated with the nonlinear medium.

3.1. Second-Order Correction. The approximate solution for the cavity problem is found using the expression for $m$ thorder correction and taking into account that the superoperator $\widehat{\mathscr{L}} \rho$ will be defined by the sum of superoperators $\widehat{J} \rho$ and $\widehat{L} \rho$; taking this into consideration, we get the second-order correction

$$
\begin{aligned}
& \rho(t) \approx e^{\widehat{S} \tau} \rho(0)+\lambda e^{\widehat{S} \tau}\left[\left(\frac{1-e^{-2 i \widehat{R} \tau}}{2 i \widehat{R}}\right) \widehat{J}+\widehat{L} \tau\right] \rho(0) \\
& +\lambda^{2} e^{\widehat{S} \tau}\left[\frac{\widehat{L}^{2} \tau^{2}}{2 !}\right] \rho(0)+\lambda^{2} e^{\widehat{S} \tau}\left[\frac{1}{2 !}\left(\frac{1-e^{-2 i \widehat{R} \tau}}{2 i \widehat{R}} \widehat{J}\right)^{2}\right. \\
& \left.+\frac{2}{2 i \widehat{R}}\left(\tau e^{-2 i \widehat{R} \tau}-\frac{1-e^{-2 i \widehat{R} \tau}}{2 i \widehat{R}} \widehat{J}\right)\right] \rho(0) \\
& +\lambda^{2} e^{\widehat{S} \tau}\left[\widehat{L} \tau\left(\frac{1-e^{-2 i \widehat{R} \tau}}{2 i \widehat{R}} \widehat{J}\right)\right] \rho(0) .
\end{aligned}
$$


The difference between (24) and (30) is that for the approximated solution $\lambda$ has been considered as a perturbation parameter. Considering as initial state a coherent state, we arrived at the approximate density matrix

$$
\rho_{\text {apro }}(\tau)=e^{-|\alpha|^{2}} \sum_{p=0}^{\infty} \sum_{q=0}^{\infty} F_{p, q}|p\rangle\langle q|,
$$

where

$$
\begin{aligned}
F_{p, q} & =\frac{\alpha^{p} \alpha^{* q}}{\sqrt{p ! q !}} e^{-i[p(p-1)-q(q-1)] \tau}\left(1+H_{p, q}\right), \\
H_{p, q} & \\
= & \frac{\lambda^{2} \tau^{2}}{2}\left[p+q-2|\alpha|^{2} e^{-i(p-q) \tau} \operatorname{sinc}[(p-q) \tau]\right]^{2} \\
& -\lambda(p+q) \tau+2 \lambda|\alpha|^{2} \tau e^{-i(p-q) \tau} \operatorname{sinc}[(p-q) \tau] \\
& -\lambda^{2}|\alpha|^{2} e^{-2 i(p-q) \tau}\left[\frac{1-e^{2 i(p-q) \tau}+2 i(p-q) \tau}{(p-q)^{2}}\right] .
\end{aligned}
$$

\section{Comparison between Exact and Approximate Solution}

A simple and direct form to visualize the evolution of a cavityKerr system in phase space is calculating a quasi-probability function. The Husimi Q-function is the simplest of all quasiprobability functions and it is defined as the expectation value of the density matrix in a coherent base $[25,26]$. Thus, if we use the exact density matrix to evaluate the $Q$-function, we find

$$
\begin{aligned}
& Q_{\text {exa }}(\beta) \\
& \quad=\frac{1}{\pi} e^{-\left(|\alpha|^{2}+|\beta|^{2}\right)} \sum_{n=0}^{\infty} \sum_{l=0}^{\infty} \frac{\left(\alpha \beta^{*}\right)^{n}\left(\alpha^{*} \beta\right)^{l}}{n ! l !} e^{-\lambda(n+l) \tau} D_{n, l},
\end{aligned}
$$

with

$$
\begin{aligned}
& D_{n, l} \\
& \quad=e^{-i[n(n-1)-m(m-1)] \tau} \exp \left\{\lambda|\alpha|^{2} \frac{1-e^{-2 \tau[\lambda+i(n-m)]}}{\lambda+i(n-m)}\right\} .
\end{aligned}
$$

For the approximated density matrix, we get

$$
\begin{aligned}
& Q_{\text {apro }}(\beta)=\frac{1}{\pi} \\
& \cdot e^{-\left(|\alpha|^{2}+|\beta|^{2}\right)} \sum_{p=0}^{\infty} \sum_{l=0}^{\infty} \frac{\left(\alpha \beta^{*}\right)^{p}\left(\alpha^{*} \beta\right)^{l}}{p ! l !} e^{-i[p(p-1)-l(l-1)] \tau}(1 \\
& \left.\quad+G_{p, l}\right)
\end{aligned}
$$

with

$$
\begin{aligned}
G_{p, l}= & \frac{\lambda^{2} \tau^{2}}{2}\left\{p+l-2|\alpha|^{2} e^{-i(p-l) \tau} \operatorname{sinc}[(p-l) \tau]\right\}^{2} \\
& -\lambda(p+l) \tau+2 \lambda|\alpha|^{2} \tau e^{-i(p-l) \tau} \operatorname{sinc}[(p-l) \tau] \\
& +4 \tau \lambda^{2}|\alpha|^{2} e^{-i(p-l) \tau} \frac{e^{-i(p-l) \tau}-\operatorname{sinc}[(p-l) \tau]}{2 i(p-l)}
\end{aligned}
$$

The Husimi function, as it evolves in time, is plotted in Figure 1 for $\lambda=0.05$. In Figure 1(a), we have the numerical results for the exact solution, and, in Figure 1(b), we have the results for the second-order correction solution. It seems that in short times the result is the same and for larger times some differences appear; in Figure 2, we show the evolution of the Husimi function for $\lambda=0.13$. Apparently, if we increase the value of lambda, the range of time in which the exact and the approximated solutions are similar becomes smaller. The reason for this behavior is easy to understand, when it is realized that the real perturbation parameter is $\lambda \tau$ and not just lambda.

As a second way to test the accuracy of the perturbation approximation, we proceed to calculate the mean photon number, which is a relevant physical quantity of the Kerr lossy cavity. Using the exact solution, we obtain

$$
\langle N\rangle_{\text {exa }}=|\alpha|^{2} e^{-2 \lambda \tau}
$$

and, with the second-order approximated solution, we get

$$
\langle N\rangle_{\text {apro }}=|\alpha|^{2}\left[1-2 \lambda \tau+\frac{(-2 \lambda \tau)^{2}}{2 !}\right]
$$

These results are presented in Figure 3. The parameters chosen are $\alpha=2$ and $\lambda=0.05,0.07,0.09,0.11$, and 0.13 . The solid lines represent the exact solution, whereas the dotted lines show the results of the perturbative solution.

The figure shows that approximate solutions with different values of $\lambda$ are indeed a good approximation for the problem of Kerr lossy cavity for short times.

Finally, as another measure of proximity for the solutions, we evaluate the distance between the exact density matrix and the approximated density matrix [27]. The geometrical measure of distance between two density matrixes is given by

$$
\left\|\rho_{1}-\rho_{2}\right\|^{2}=\frac{1}{2} \operatorname{Tr}\left(\rho_{1}-\rho_{2}\right)^{2}=1-F
$$

where $F=\operatorname{Tr}\left(\rho_{1} \rho_{2}\right)+(1 / 2)\left(2-\operatorname{Tr} \rho_{1}^{2}-\operatorname{Tr} \rho_{2}^{2}\right)$ is a parameter that evaluates the closeness of $\rho_{1}$ and $\rho_{2}$. Both matrices will be similar if $F=1$ or completely different if $F=0$. 

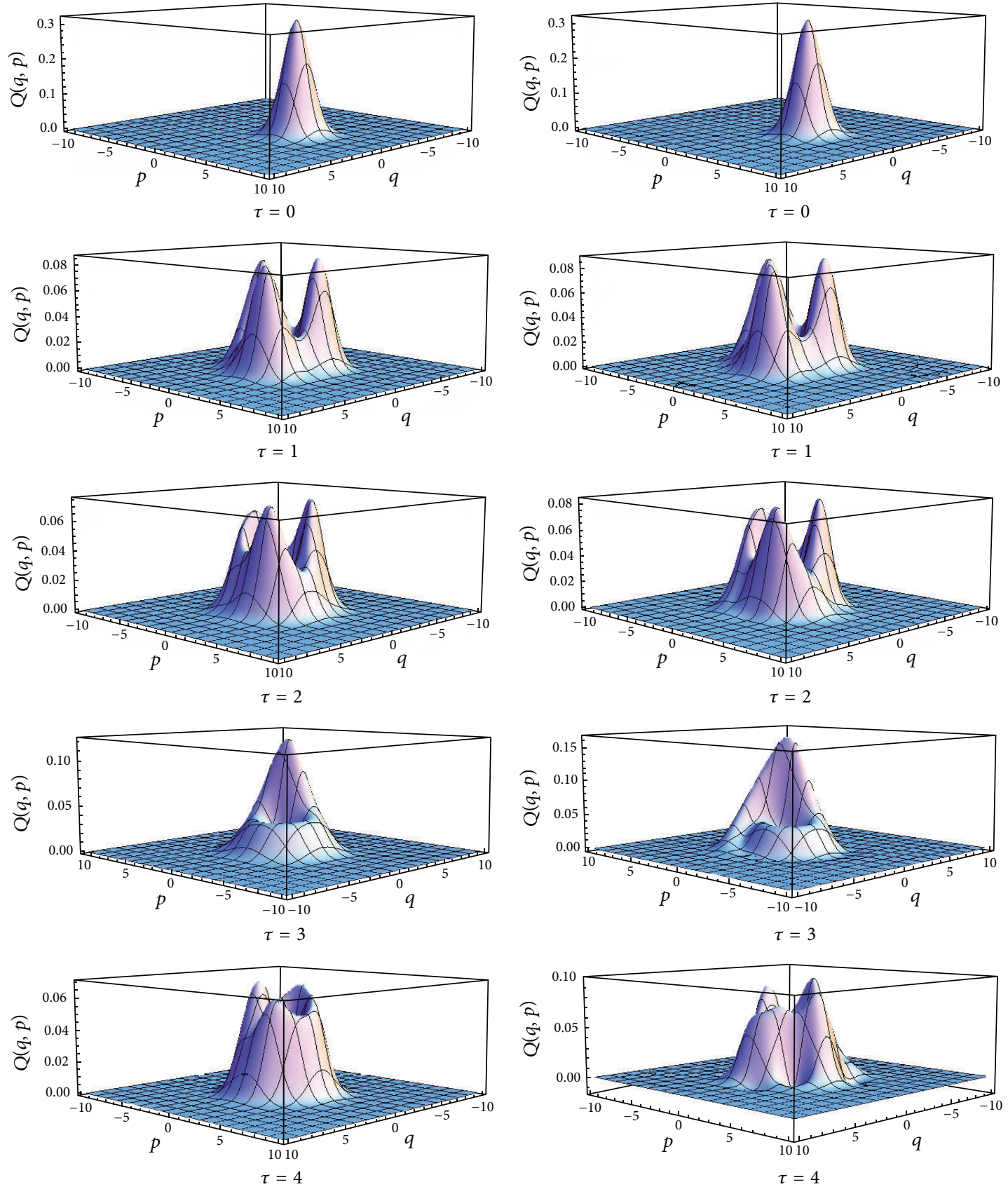

(a)

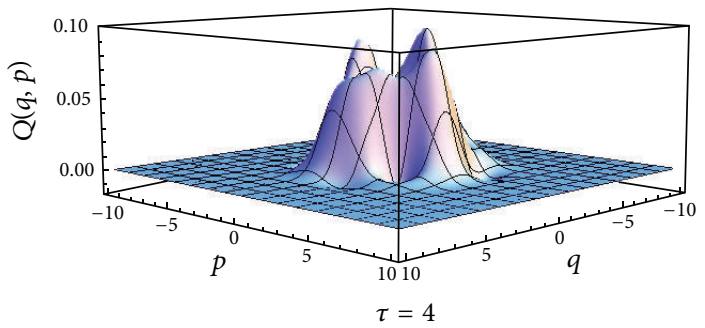

(b)

FIGURE 1: Evolution of the $Q$-function with $\alpha=2$ and $\lambda=0.05$. (a) Exact solution and (b) approximated solution.

Figure 4 illustrates the numerical evaluation of parameter $F$ upon $\tau$, for $\alpha=2$ and different values of $\lambda$. This plot shows that density matrices are very similar for short times; these results coincide and are in agreement with the temporal behavior of the Q-function and the average number of photons.

\section{Conclusions}

In summary, we can conclude that in the examined case, a lossy cavity filled with a Kerr medium, the matrix perturbative method gives good results. When time grows the results start to differ, but that is not surprising since the real measure of the perturbation is not given by only $\lambda$, and it is given by the product $\lambda \tau$. The same logical behavior is observed in the case of the Husimi function and the parameter $F$ for different values of lambda, where we found good agreement for short times and some differences when the time grows. The second-order result is enough to precisely reproduce the exact solution for this specific system; when studying more complicated systems, the contributions of orders superior to the first one could be relevant. 

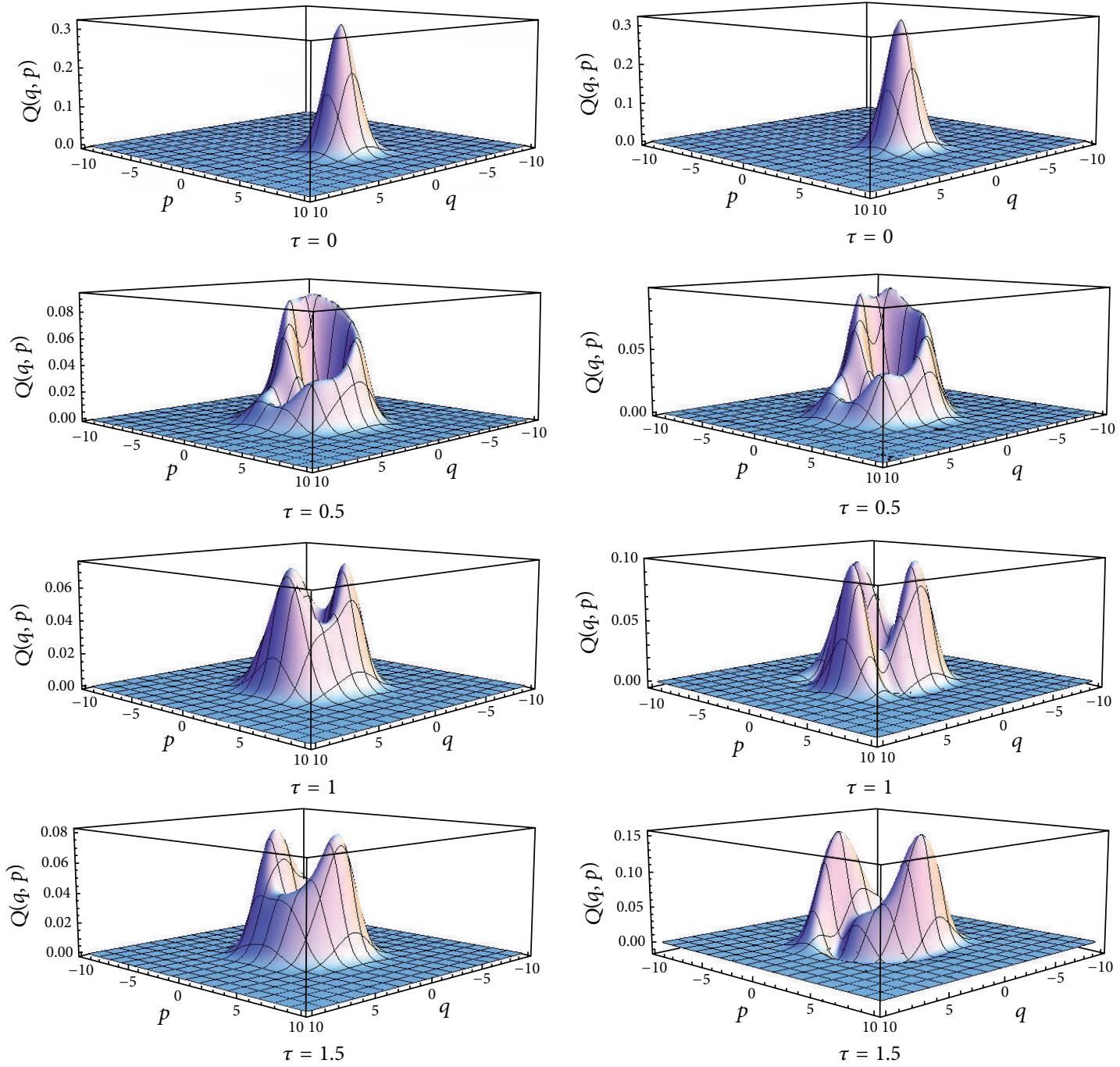

(a)

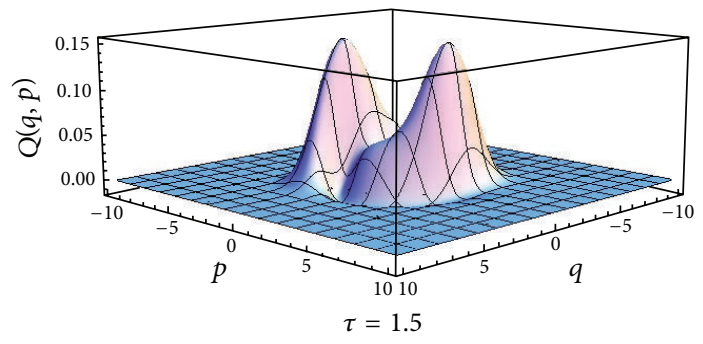

(b)

Figure 2: Evolution of the Q-function with $\alpha=2$ and $\lambda=0.13$. (a) Exact solution and (b) approximated solution.

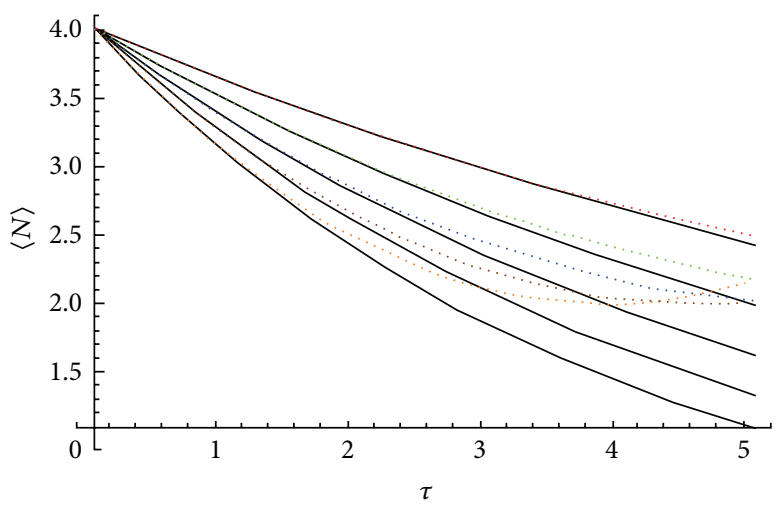
$\square \lambda=0.13$
$\square \lambda=0.07$
$\square \lambda=0.11$
$\square \lambda=0.05$

Figure 3: The mean photon number $\langle N\rangle$ versus time $\tau$, with $\alpha=2$ and $\lambda=0.05,0.07,0.09,0.11$, and 0.13 . The solid lines are for the exact solution, while the dotted lines are for the perturbative solution. 


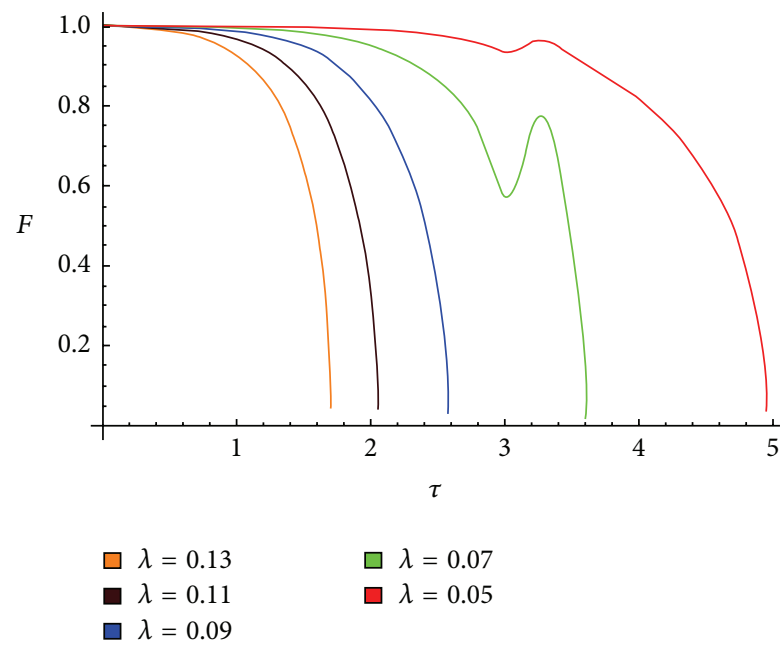

Figure 4: Plot of $F=\operatorname{Tr}\left(\rho_{1} \rho_{2}\right)+(1 / 2)\left(2-\operatorname{Tr} \rho_{1}^{2}-\operatorname{Tr} \rho_{2}^{2}\right)$ versus $\tau$ for different values of $\lambda$.

\section{Competing Interests}

The authors declare that they have no competing interests.

\section{Acknowledgments}

B. M. Villegas-Martínez acknowledges CONACYT for support.

\section{References}

[1] M. Gell-Mann and J. B. Hartle, "Quantum mechanics in the light of quantum cosmology," in Complexity, Entropy, and the Physics of Information, W. H. Zurek, Ed., Addison-Wesley, Reading, Mass, USA, 1990.

[2] M. Gell-Mann and J. B. Hartle, "Classical equations for quantum systems,” Physical Review D, vol. 47, no. 8, pp. 3345-3382, 1993.

[3] B. L. Littre and A. Matacz, "Quantum noise in gravitation and cosmology," in Proceedings of the International Workshop on Fluctuations and Order: A New Synthesis, Los Alamos, NM, USA, 1993.

[4] W. G. Unruh, "Sonic analogue of black holes and the effects of high frequencies on black hole evaporation," Physical Review D, vol. 51, no. 6, pp. 2827-2838, 1995.

[5] F. Petruccione and H.-P. Breuer, The Theory of Open Quantum Systems, Oxford University Press, 2002.

[6] A. O. Caldeira and A. J. Leggett, "Quantum tunnelling in a dissipative system," Annals of Physics, vol. 149, no. 2, pp. 374-456, 1983.

[7] D. F. Walls, M. J. Collet, and G. J. Milburn, "Analysis of a quantum measurement," Physical Review D, vol. 32, no. 12, pp. 3208-3215, 1985.

[8] B. L. Hu, J. P. Paz, and S. Sinha, "Minisuperspace as a quantum open system," in Directions in General Relativity. Proceedings of the 1993 International Symposium, Maryland: Papers in Honor of Charles Misner. Volume 1, B. L. Hu, M. P. Ryan Jr., and C. V. Vishveshwara, Eds., pp. 145-165, Cambridge University Press, New York, NY, USA, 1993.

[9] Y. Yamamoto and I. Atas, Mesoscopic Quantum Optics, John Wiley \& Sons, New York, NY, USA, 1999.
[10] S. Attal, A. Joye, and C.-A. Pillet, Eds., Open Quantum Systems I: The Hamiltonian Approach, vol. 1880 of Springer Science and Business Media, Springer, 2006.

[11] E. B. Davies, "Markovian master equations," Communications in Mathematical Physics, vol. 39, no. 2, pp. 91-110, 1974.

[12] V. Gorini, A. Frigerio, M. Verri, A. Kossakowski, and E. C. Sudarshan, "Properties of quantum Markovian master equations," Reports on Mathematical Physics, vol. 13, no. 2, pp. 149173,1978

[13] A. B. Klimov and J. L. Romero, "An algebraic solution of Lindblad-type master equations," Journal of Optics B: Quantum and Semiclassical Optics, vol. 5, no. 3, pp. S316-S321, 2003.

[14] T. Prosen, "Third quantization: a general method to solve master equations for quadratic open Fermi systems," New Journal of Physics, vol. 10, no. 4, Article ID 043026, 23 pages, 2008.

[15] L. M. Arévalo-Aguilar and H. Moya-Cessa, "Solution to the master equation for a quantized cavity mode," Quantum and Semiclassical Optics B, vol. 10, no. 5, pp. 671-674, 1998.

[16] Z.-J. Zhang, D.-G. Jiang, and W. Wang, "Perturbation theory for open two-level nonlinear quantum systems," Communications in Theoretical Physics, vol. 56, no. 1, article 12, pp. 67-70, 2011.

[17] X. X. Yi, C. Li, and J. C. Su, "Perturbative expansion for the master equation and its applications," Physical Review A-Atomic, Molecular, and Optical Physics, vol. 62, article 013819, 2000.

[18] J. Kim II, M. C. Nemes, A. F. R. de Toledo Piza, and H. E. Borges, "Perturbative expansion for coherence loss," Physical Review Letters, vol. 77, no. 2, pp. 207-210, 1996.

[19] H. Moya-Cessa, J. A. Roversi, S. M. Dutra, and A. VidiellaBarranco, "Recovering coherence from decoherence: a method of quantum-state reconstruction," Physical Review A-Atomic, Molecular, and Optical Physics, vol. 60, no. 5, pp. 4029-4033, 1999.

[20] H. Moya-Cessa, S. M. Dutra, J. A. Roversi, and A. VidiellaBarranco, "Quantum state reconstruction in the presence of dissipation," Journal of Modern Optics, vol. 46, no. 4, pp. 555$558,1999$.

[21] H. M. Moya-Cessa, F. Soto-Eguibar, J. M. Vargas-Martínez, R. Juárez-Amaro, and A. Zúñiga-Segundo, "Ion-laser interactions: the most complete solution," Physics Reports, vol. 513, no. 5, pp. 229-261, 2012.

[22] H. M. Moya-Cessa and F. Soto-Eguibar, Introduction to Quantum Optics, Rinton Press, Paramus, NJ, USA, 2011.

[23] J. Martínez-Carranza, F. Soto-Eguibar, and H. Moya-Cessa, "Alternative analysis to perturbation theory in quantum mechanics: dyson series in matrix form," European Physical Journal $D$, vol. 66, no. 1, article 22, 2012.

[24] L. M. Arévalo-Aguilar, R. Juárez-Amaro, J. M. Vargas-Martínez, O. Aguilar-Loreto, and H. Moya-Cessa, "Solution of master equations for the anharmonic oscillator interacting with a heat bath and for parametric down conversion process," Applied Mathematics \& Information Sciences, vol. 2, no. 1, pp. 43-49, 2008.

[25] W. P. Scheleich, Quantum Optics in Phase Space, Wiley-VCH, Berlin, Germany, 2001.

[26] R. Loudon, "Coherent states: applications in physics and mathematical physics," Optica Acta: International Journal of Optics, vol. 33, no. 3, p. 219, 1986.

[27] S. N. Filippov and V. I. Man'ko, "Geometrical interpretation of the density matrix: mixed and entangled states," Journal of Russian Laser Research, vol. 29, no. 6, pp. 564-580, 2008. 


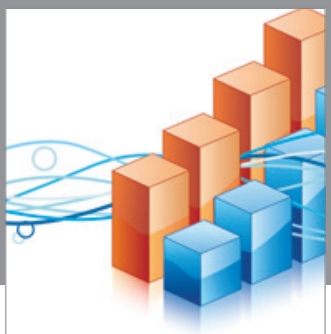

Advances in

Operations Research

vatem alat4

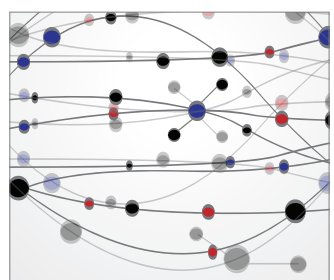

\section{The Scientific} World Journal
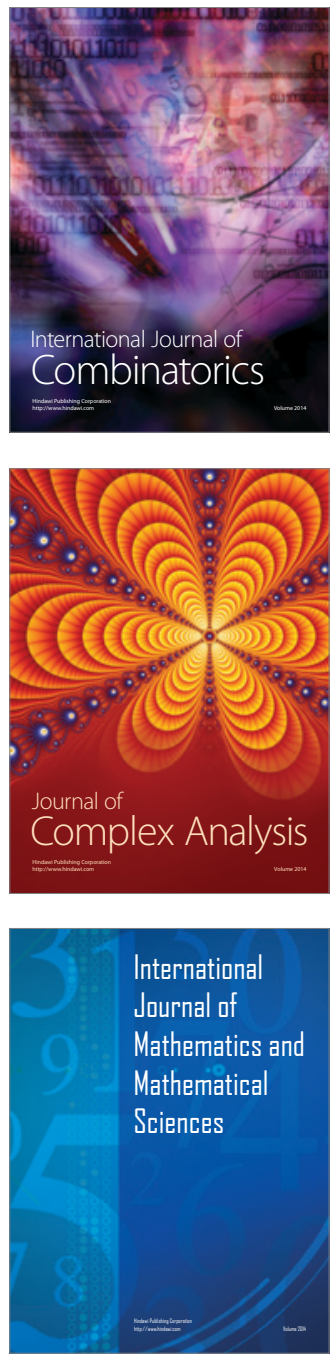
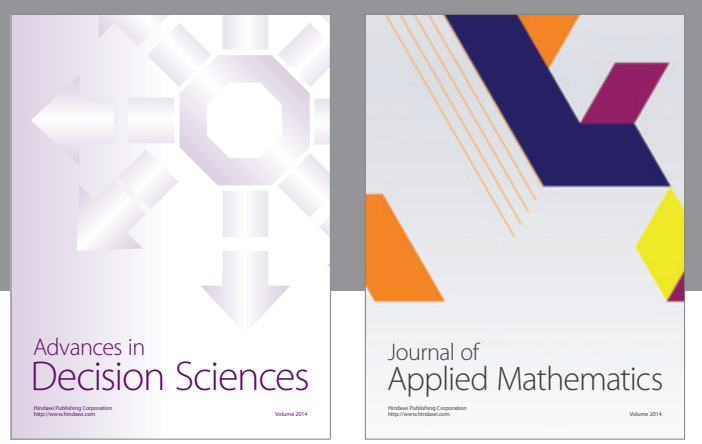

Algebra

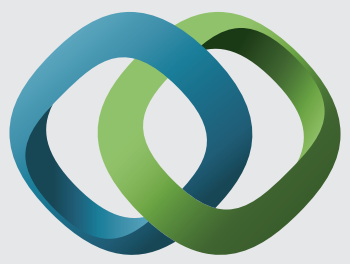

\section{Hindawi}

Submit your manuscripts at

http://www.hindawi.com
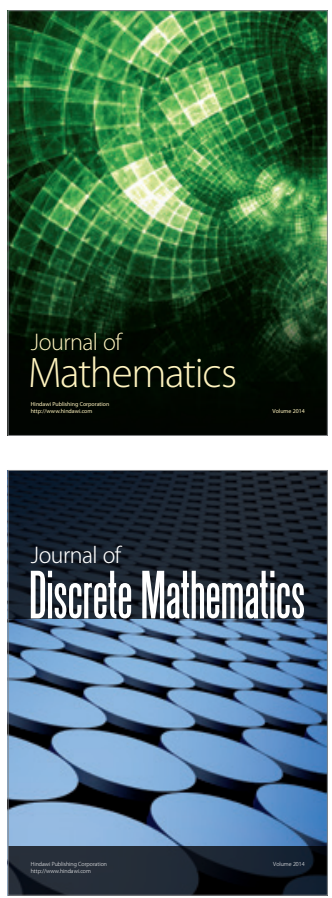

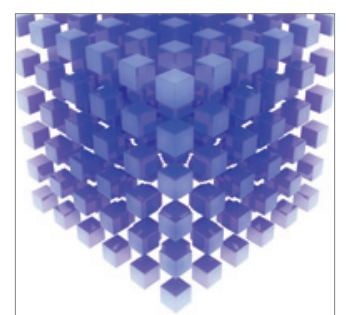

Mathematical Problems in Engineering
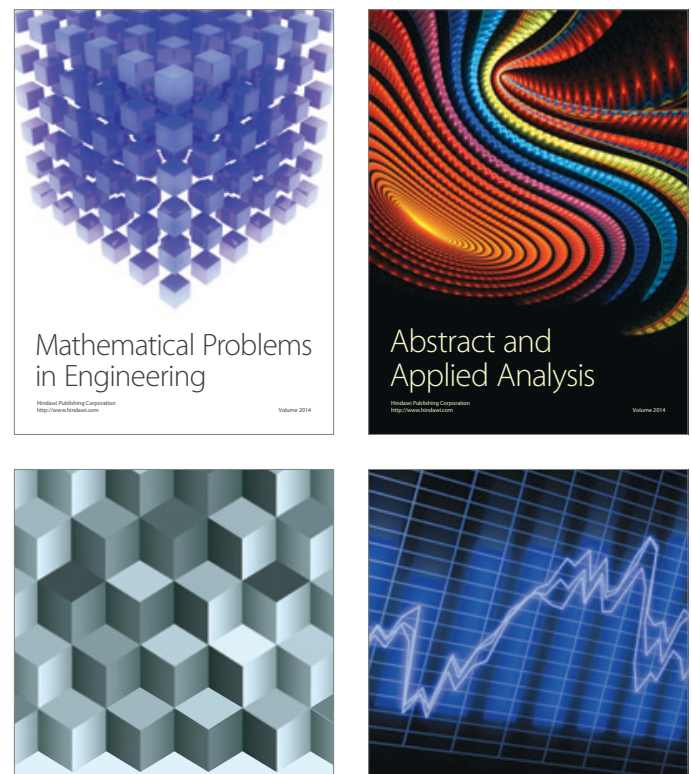

Journal of

Function Spaces

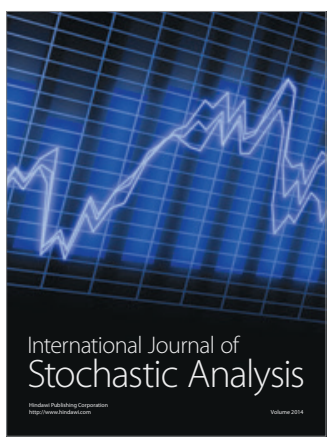

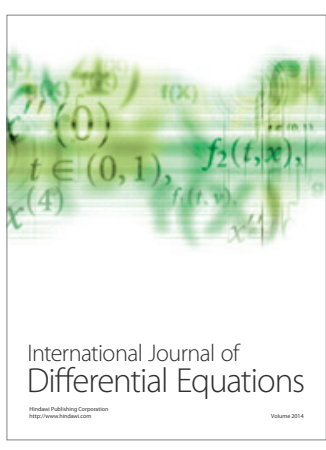
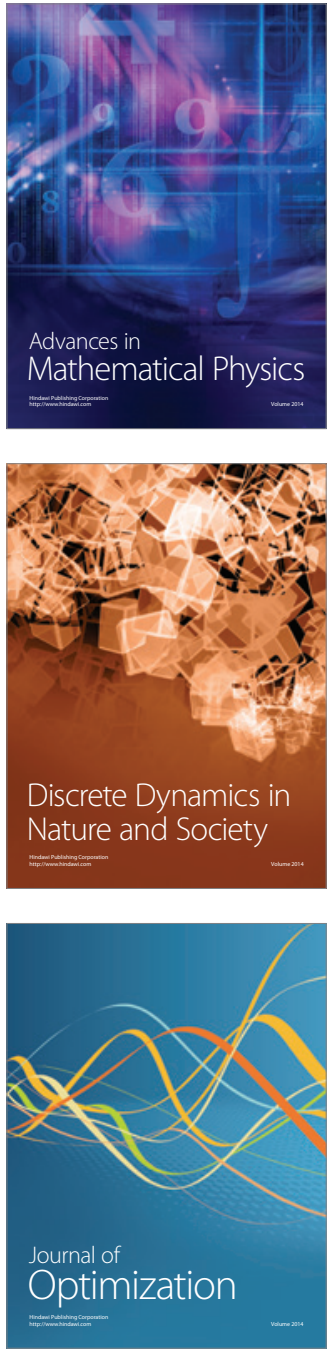